\title{
A Robust and Scalable Pareto Optimal Ball Passing Algorithm for the Robotic Soccer
}

\author{
Vadim Kyrylov \\ Rogers State University
}

USA

\begin{abstract}
We are looking for a generic solution for the optimized ball passing problem in the robotic soccer which is applicable to different RoboCup leagues and other digital simulated sports games like basketball or ice hockey. In doing so, we show that previously published ball passing methods do not properly balance the anticipated rewards, costs, and risks. The multi-criteria nature of this optimization problem requires using the Pareto optimality approach. As the problem itself is substantially inconvex, nothing else except the search of all available alternatives in the Pareto set appears to be applicable in this case. Real-time constraints are further complicating the problem. We propose a scalable and robust solution for decision making with multiple optimality criteria; its quality degrades in a graceful way once the real time constrains are kicking in. Our method is treating equally direct and leading passes to the partners and self passing while fast dribbling the ball by the player. The $\varepsilon$ new method also allows easily modeling the whole spectrum of risk aversive to risk talking O attitudes; therefore it is generic indeed.
\end{abstract}

\section{Introduction}

\subsection{Ball Passing Algorithms: State of the Art}

In the real-life and robotic soccer the rational player who controls the ball has four choices: shooting at the opponent goal, dribbling (moving without losing control of the ball), holding the ball, and passing it to a teammate or to self.

We are assuming that at given moment of time the choice between these higher-level options has been already made and the player is going to pass the ball by making the further choice between lower-level options representing alternative ways to execute this pass. As this decision making skill is generally regarded to be critical for the success of a soccer team, we investigate how ball passing could be implemented in the best possible way.

Early RoboCup scholars have identified the major peculiarities of ball passing and developed algorithms for the simulated soccer $[1,2]$. These methods have proved to be good, as Ф the soccer teams using them have been performing in the RoboCup competitions very successfully. Because we have not found in the academic literature any other published meth-

Source: Robotic Soccer, Book edited by: Pedro Lima, ISBN 978-3-902613-21-9, pp. 598, December 2007, Itech Education and Publishing, Vienna, Austria 
ods since then, this is presumably the state of the art; thus in what follows, we will be using these two algorithms as the prototypes for further improvement.

In both algorithms the soccer agent chooses values of the direction of the kick and its force. In [1] the anticipated outcome of this action is evaluated using two heuristic indicators: (1) the tactical value of the end point where the ball can be intercepted by the fastest teammate and (2) the fuzzy estimate of the likelihood of this interception. The latter is the function of the time balance between the fastest teammate to the ball and the fastest opponent. Stone \& McAllister [1] also proposed using both direct passes to the receiver's position and leading passes that allow sending the ball to the teammate who is blocked by opponents from receiving a direct pass. In this method, the tactical value of the interception point is the only criterion for selecting the best option; the likelihood of success is used as a constraint for eliminating poor alternatives. Although this method has proved to be good enough for ATT-CMUnited-2000 making it to the third place in the RoboCup 2000 competition in the simulation league, it neglects some risks that are indeed present in the soccer game. One of them is the possible proximity of other opponents to the anticipated interception point. We would also disagree with [1] in that the ball should be always intercepted by the receiver in the minimal time. This may result in lost opportunities in executing leading passes when the ball is deliberately sent to the point of the field having the best tactical value. Thus the receiving player must be moving exactly towards this point if it is indeed safe to do so; the ball passing algorithm should be based on this assumption.

The ball passing decision making algorithm implemented in FC Portugal, the 2000 World champion [2], in many respects is similar to [1]. Additionally it is taking into account the opponent player congestion in the vicinity of the ball destination. Several additional factors are considered, such as ball travel distance, opponent goal scoring opportunity if the pass is successful, and the possible outcomes if the ball would not be intercepted as intended. The decision is made by deliberating on 5 options for each receiving teammate: direct pass, leading pass, pass to the expected location of the teammate, pass to a point near teammate having low congestion, and pass along a low congestion line. Each alternative is evaluated using 9 performance indicators. With the purpose of making a choice, these indicators are analyzed using a set of heuristic logical rules, i.e. a sort of a decision tree. It is known that FC Portugal outplayed ATT-CMUnited-2000 in the 2000 RoboCup competitions. We can only speculate whether the improved ball passing algorithm was a decisive factor or not. It is clear that the second algorithm must be good; yet it is much more complex and more difficult to analyze and optimize.

A presumably even more advanced ball passing algorithm incorporating leading passes with player collaborating using inter-player communication was recently reported in a very short paper [3]. This algorithm is all based on a decision tree. In what follows, we demonstrate that a decision tree may overlook some indeed good ball passing options, which is the shortcoming of this algorithm.

\subsection{Unresolved Issues and Research Objectives}

We would mention three issues that we address in this paper.

1. No benchmark so far. Although known algorithms have proved to be good indeed, from the scholarly standpoint their common weakness is in that they are just collections of sophisticated heuristics; it is still unknown to what extent they could be further improved and what the benchmark solution is. 
2. Smoothly balancing rewards and risk. We believe that the ability to easy implement a continuous spectrum of risk-taking vs. risk aversive strategies by the soccer agent is highly desirable feature in any algorithm for modeling player behavior. Indeed, while risky passes should be completely avoided near own goal, they are affordable or even could be desirable on the opponent side. However, in the existing ball passing methods the deliberations with regards to risks and rewards, if any, do not render themselves as a controlled feature.

3. Avoiding possible conflicts with the real-time constraints, as the ball passing algorithms are computation intensive. So far the RoboCup scholars have been tackling with this inventively, yet somewhat unsystematically, by reducing computations to the reasonable minimum just by using various heuristics. Further reduction in the amount of required computations in such algorithms may be normally possible at the expense of abrupt loss in the quality of decisions, as this is done, for example, by removing some branches in the decision tree.

We believe that these issues can be resolved and it is indeed possible using the Decision Science techniques, such as multi-criteria decision analysis (MCDA). In doing so, we are pursuing the following objectives.

Developing a theoretical framework for a truly optimal ball passing algorithm that is utilizing all the potential of the soccer agent and could serve as a benchmark. We want this solution to be generic and thus reusable in any robotic soccer league. This intention is standing in a concert with other RoboCup scholars looking for generic solutions [4].

Fully identifying rewards, risks, and costs involved in passing the ball and demonstrating how they could be balanced in the proposed framework. In particular, we wish to offer an easy way to implementing a continuous spectrum of attitudes by the soccer player. We also want that decisions about ball passes to stem from the deliberation of all reasonable options that are available to the moment with very few parameters controlling the balance between risk taking and risk aversion. These parameters must all have clear meaning.

All-in-one implementation. We would like to have a generic solution that subsumed direct and leading passes and passes to self. With regards to leading passes, too few details have been revealed in both [1] and [2] so far on how the end point is determined, although this is a non-trivial task. We wish to propose a simple method for implementing this critical feature.

Addressing the real-time constraints. We want to propose a truly scalable solution with just one parameter which determines the amount of the required computations. We also want to design a robust ball passing algorithm so that if we are forced to eliminate some computations for the sake of saving computation time, that would be resulting only in a gradual loss of the decision quality.

Section 2 provides the reward/risk/cost analysis of ball passing. In Section 3 we briefly explain the essence of the Pareto optimality and demonstrate how this concept applies to optimized ball passing. Section 4 provides a solution to searching the Pareto set for the optimal decision. Section 5 explains how the real-time constraints in the proposed method could be walked around in a graceful way. Section 6 concludes this work. 


\section{Rewards, Risks, and Costs in Ball Passing}

Prior to developing the optimal decision making algorithm, in this section we identify the criteria that govern the decision by the soccer player to pass the ball; we wish this list to be as complete as possible.

In doing so, we slightly modify the ball passing problem formulation as compared to [1, 2]. In our case, player with the ball considers all possible points $(x, y)$ in the field and must decide to which point he should send the ball now; he also must choose the speed that the ball will have upon the arrival in this point. The end speed affects the probability of the successful interception by the receiving teammate; it also determines the ball travel time and thus the incurred risk. The decision is made by comparing these and some other criteria calculated for different ball passing options.

Once the passing player has made his choice of the point and of the ball end speed, he is able to determine the kicking force and direction, which are the actual decision variables. If the required kicking force exceeds the available limit, the point is just removed from the consideration. Likewise, points are eliminated if the perceived risks are prohibitively high. This feature could be implemented similar to the existing algorithms; conservative elimination rules can be applied to avoid unnecessary deliberations and reduce computations. In what follows, we demonstrate this on examples.

Each remaining potential destination point for pass is assigned a vector criterion having continuous values of its $m$ components. So there is a two-dimensional decision space (kicking_force, direction) and an $m$-dimensional criterion space.

For the analysis, we replace the continuous decision space by a discrete one. Initially, with the purpose to find a benchmark solution, we deliberately consider that the number of points in this space is large enough so that in terms of our criteria the difference between the neighbors was negligibly small.

For the further analysis of the decision criteria semantics, we split them in three categories: (1) rewards, (2) risks, and (3) costs.

Rewards. We see two rewards, or gains, from passing the ball; we wish to maximize both. First can be measured by the tactical value $f_{1}(x, y)$ of the point where the ball will be sent to. As the tactical value proposed in [1] and [3] serves this purpose very well, we will be using a similar one. It encourages sending the ball up the field to the opponent side; points closer to the opponent goal have higher tactical value. Because of the function is anti-symmetrical for $x$-coordinate, it equally discourages from passing the ball close to own goal.

The second reward function reflects the chance to score the opponent goal once the ball arrives to its destination $(x, y)$. This could be measured by the following heuristic criterion:

$$
f_{2}(x, y)=\alpha(x, y) /\left(n_{\text {opp }}(x, y)+1\right)
$$

where $\mathrm{\alpha}(x, y)$ is the angular size of the goal as seen from $(x, y)$ and $n_{\text {opp }}(x, y)$ is the anticipated number of opponent players in the cone having $(x, y)$ as its vertex; the base of this cone is the opponent goal that is slightly stretched sideways.

Risks. The risks involved in ball passing can be all regarded as soft constraints because of presence of uncertainty; this is a further improvement of the idea proposed in [1]. Risks were identified bearing in mind that the receiving player is intercepting the ball in minimal time if only he has no other choice; rather we assume that he must be moving to the point where the sender decided to get it delivered to. (This could be implemented using communication and/or so-called 'smart' ball interception algorithm.) Hence we need to address more risk factors than our predecessors. If the risk incurs any time balance, we calculate it 
with some conservative margin in the favor of the opponents. This margin increases with the distance to the ball passing end point.

1. Opponent may reach $(x, y)$ before the teammate. The risk function $r_{1}(x, y)$ is the time balance between the arrivals of the fastest opponent and the fastest teammate to this point. If this balance is negative, $r_{1}(x, y)$ is zero.

2. Ball can be intercepted by the opponent on its way to $(x, y)$. The risk function $r_{2}(x, y)$ is the time balance between the intended arrival time of the ball in $(x, y)$ and the earliest time when it can be stolen by the opponent. If this balance is negative, $r_{2}(x, y)$ is zero.

3. Teammate may be too late in point $(x, y)$ after the ball rolls by. So the risk function $r_{3}(x, y)$ is the probability of the teammate failing to reach the ball in $(x, y)$. This probability is 1 if the teammate cannot arrive in $(x, y)$ before the ball and decreases fast to 0 with the extra time available for the player. The risk also increases if the ball is moving in $(x, y)$ too fast which is making it difficult to intercept.

4. Once the ball has been received by the teammate, too many opponents may get close by. The risk function $r_{4}(x, y)$ is the time balance between the arrivals of the first and second fastest opponents in $(x, y)$, plus some positive heuristic constant $r_{4 \max }$. If this sum is negative, $r_{4}(x, y)$ is zero. If both opponents arrive simultaneously, $r_{4}(x, y)=r_{4 \max }$.

5. If the teammate fails to intercept the ball, it may cross the field boundary; the interruption of the game by the referee that would follow thus giving some advantage to the opponent. Similar case is when passing the ball may create the offside situation. The risk function $r_{5}(x, y)$ is zero everywhere except the points where the ball trajectory, if continued, may intersect the field boundary or the offside line. In these cases the risk is some constant $r_{5 \max }$ less the time remained until the ball crosses the line. Thus on the field boundaries we get $r_{5}(x, y)=r_{5 \max }$. Negative values are brought up to zero.

6. The receiving player may have too low stamina to chase and handle the ball (this information is made available to the ball controller via aural sensor). The risk function $r_{6}(x, y)$ is the time when the receiving teammate reported low stamina minus current time plus positive heuristic constant $r_{6 \max }$. If this sum is negative, $r_{6}(x, y)$ is zero.

7. Ball may not reach the destination point at all, as $(x, y)$ is too far away for given initial ball speed. As the ball movement is distorted by noise, the actual maximal ball traveling distance may differ from the calculated theoretical one, $D_{\max }$. A soft constraint $r_{7}(x, y)$ is used to reflect this risk. For points whose distance is significantly less than $D_{\max }, r_{7}(x, y)$ is zero; near $D_{\max }$ values of $r_{7}(x, y)$ are positive.

For convenience, the seven risk functions described above might be scaled so that they all are taking values in, say $(0,10)$. We wish to simultaneously minimize each of them.

Costs. The obvious cost factor is the time required for obtaining the anticipated rewards, which we want to minimize. Taking this in consideration makes sense because the precision of the situation prediction decreases with the forecast time substantially. In this respect, it is just another risk factor. Yet cost is also may be an objective, as we are obviously interested in achieving the tactical gain faster. Cost could be measured by the nonlinear function $c_{1}(x, y)$ whose value is zero for short passes and grows with the time needed the ball to reach $(x, y)$. This criterion would be discouraging too long passes if, given all the rest conditions equal, shorter ones exist. 
Discussion. We believe that this is the most complete list of factors affecting ball passing, as it contains new elements. Heuristic constants are used for scaling only; thus they are not the 'magic numbers' that are omnipresent in existing ball passing algorithms. Some factors have been taken into account by previous scholars, yet none of them has provided a comprehensive list in academic publications. The identified risks have different severity and likelihood to occur. Of them, risk factor 1, is probably best known to all RoboCup scholars, as it is incurred very often. On the other hand, factor 7, although well understood, has been presumably treated in $[1,2]$ just as a hard constraint. However, a soft constraint is more applicable in the general case as the ball movement noise may be significant in the RoboCup leagues dealing with physical robots. As in $[1,2]$ the interception of the ball only in minimal time was assumed, risk factor 2 was not considered. By deliberately distinguishing between risks 1 and 2, we thus propose more general approach. Risk factor 6 has been also left unnoticed in the RoboCup literature; in some situations, however, this may result in the complete failure to pass the ball. Also new is the cost consideration; it looks like nobody before has tried to consider the cost as a factor. Yet the most significant difference of our approach from $[1,2]$ is in that we are not applying any logical rules to the performance indicators, nor do we suggest merging them in one.

Concept demonstration. With the sole purpose of the concept demonstration used throughout this paper, we have designed an example with three simplifications. (1) Decision space is further reduced to determining the direction of pass only; end speed in the destination point $(x, y)$ is a fixed parameter of the algorithm. (2) Only the tactical value of the end point is used as the reward. (3) Risk and cost factors merged in just one parameter by applying heuristic rules.

This allowed using two-dimensional displays for the visualization. The full-blown algorithm is treating all criteria separately without merging those using heuristics.

Screenshots in Fig.1 illustrate application of different constraints to a grid of 3600 points considered as candidates for passing the ball by player 11 from the right-hand team. The eliminated points are shown in light gray; the darker points are the remaining alternatives. The player must select the best from them based on the vector of performance indicators available for each point.

We wish this decision to be optimal in some sense. This sense is the Pareto optimality.

\section{Applying the Pareto Optimality Principle to Ball Passing}

Pareto optimality, first originated in economics, is now a standard principle for solving vector optimization problems with conflicting criteria $[5,6]$. The criteria identified in Section 2 are indeed conflicting, as the higher rewards are normally coming at higher risk and cost. In what follows, for the purpose of illustration, we will replace the reward function with the negation thereof; thus we want all our criteria to be minimized simultaneously. In the general case, though, simultaneous minimization cannot be achieved. The Pareto optimality principle only offers a method for substantially reducing the set of decision alternatives by identifying among them the set of so-called non-dominated alternatives; altogether they are making the Pareto set, or the Pareto frontier $[5,6]$.

By definition, the criteria vector $\mathbf{v}_{i}=\left\{v_{\mathrm{i} 1}, \ldots, v_{\mathrm{im}}\right\}$ is dominated by vector $\mathbf{v}_{j}=\left\{v_{\mathrm{j} 1}, \ldots, v_{\mathrm{jm}}\right\}$ if the following condition holds:

$$
\forall k\left\{v_{i k}>v_{j k}\right\}
$$




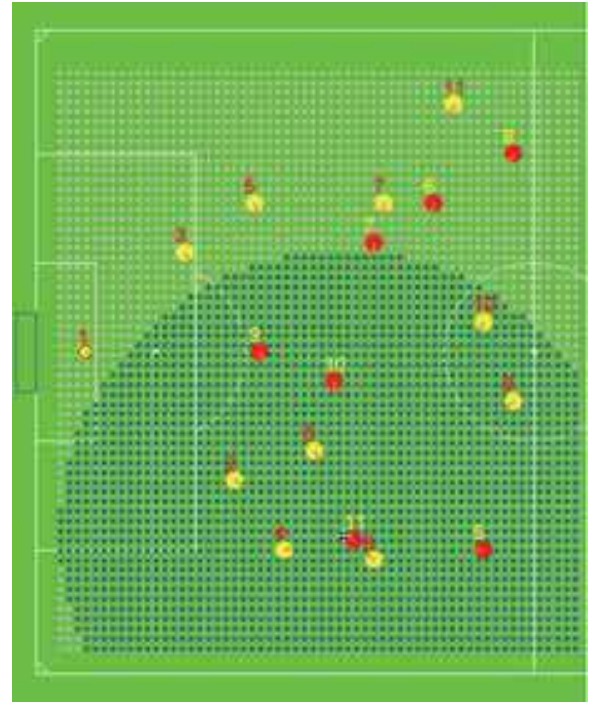

(a) applied the maximal ball rolling distance constraint only (risk factor 7)

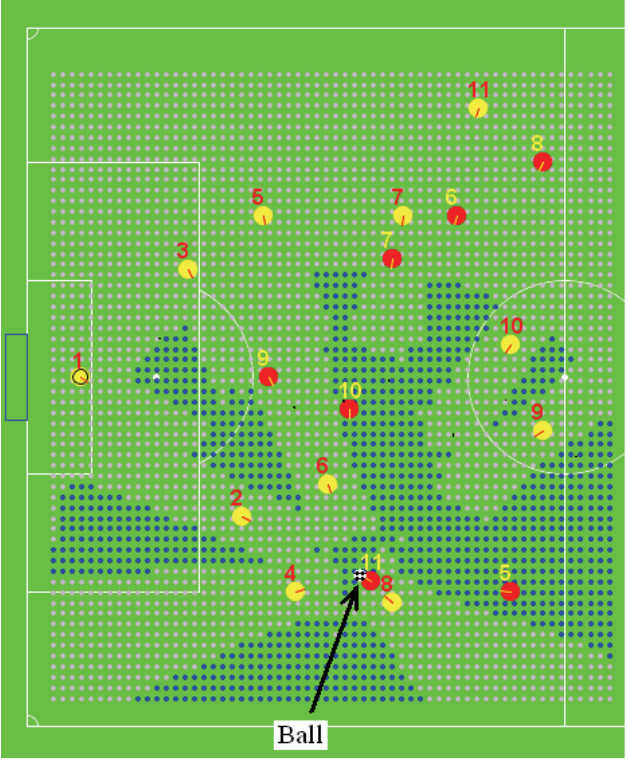

(c) added the early interception of the ball by the opponent (risk factors 2 and 7)

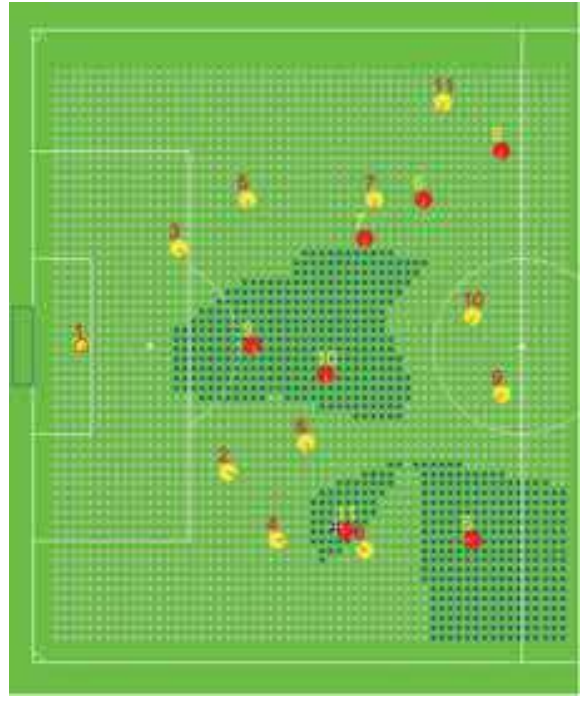

(b) added the opponent/teammate time balance constraint (risk factors 1 and 7)

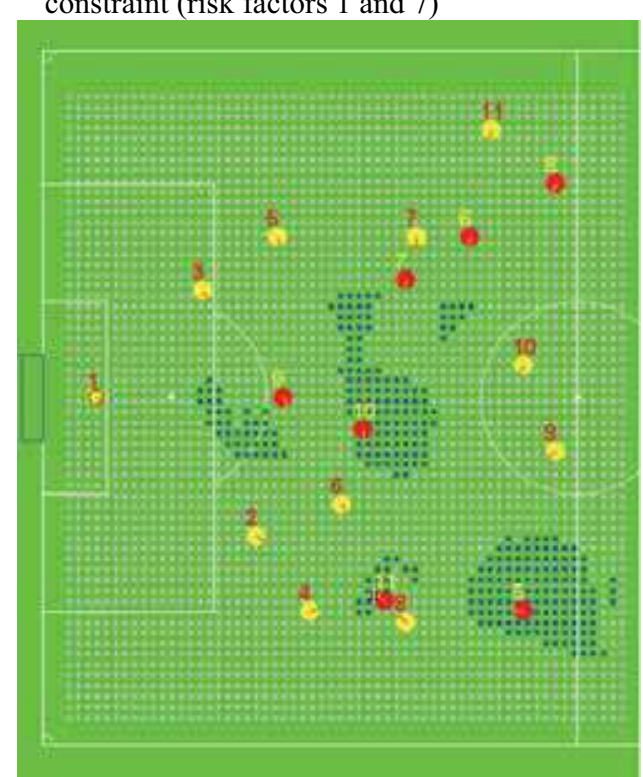

(d) all risk constraints applied; the remaining points are the player decision making options

Fig.1. Screenshots of the software tool developed for analyzing the soccer player tactics. Of the original 3600 points, some have been eliminated by applying the ball distance constraint first. Individual captions explain additional constraints that have been applied then 
This means that $\mathbf{v}_{j}$ is located inside the cone in $\mathrm{Rm}$ with the vertex $\mathbf{v}_{i}$, the sides of this cone being parallel to the coordinate subspaces $\mathrm{R}^{\mathrm{m}-1}$. Fig. 2 illustrates this situation for $m=2$.

By definition, the Pareto set is the subset of the alternatives which are all non-dominated. An example is shown in Fig. 3. Note that the Pareto set is not necessarily convex, nor is it in the general case even connected. The computational complexity of determining the Pareto subset in the finite set with $N$ elements is $O\left(N^{2}\right)$.

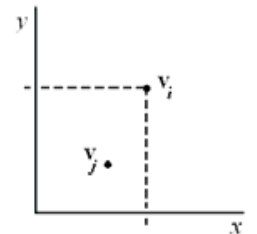

Fig.2. Vector $\mathbf{v}_{j}$ dominates $\mathbf{v}_{i}$

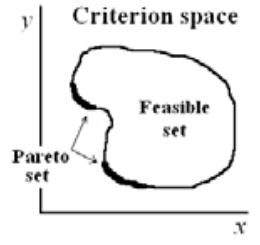

Fig.3. The Pareto set contains all non-dominated points

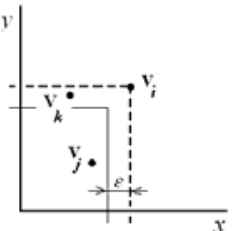

Fig.4. While $\mathbf{v}_{j}$ both dominates and $\varepsilon$ dominates $\mathbf{v}_{i}, \mathbf{v}_{k}$ dominates $\mathbf{v}_{i}$ only plainly

In Section 4, we will be also using a weakened version of the dominance relation, which is called $\varepsilon$-domination (Fig.4). The set of non- $\varepsilon$-dominated points is referred to as $\varepsilon$-Pareto set. The common-sense meaning of a non-dominated alternative $\mathbf{v}_{j}$ is that outside the Pareto set there is no another alternative that outperforms $\mathbf{v}_{j}$ simultaneously by all criteria; at least one criterion value is worse, anyway. From this follows that the optimal decision should be sought within the Pareto set; all the rest alternatives could be just eliminated as they are all inferior.

Noteworthy that, eliminating decision alternatives before identifying the Pareto set, as it has been done before in the ball passing algorithms, may result in that some of the Pareto optimal points would be apparently removed without even evaluating thereof. This is exactly what may happen in decision trees. Unless the tree decision conditions are designed so carefully that any eliminations do not affect the Pareto set, there is no guarantee that the decision making algorithm yields optimal solution to the problem in all cases. However, the trouble is in that such a decision tree is difficult to design, and for each new applied problem this must be done over and over again. On the other hand, the Pareto optimality principle offers a general solution.

So in ball passing we follow this principle. The Pareto set of the alternatives that player 11 in Fig.1(d) should be indeed choosing from is shown in Fig 5. As all the rest alternatives are dominated, we should eliminate them. This example suggests that either the leading pass to player 9 should be executed (five slightly different options), or player 11 should leave the ball for himself (two options). Passes to player 10 are not in the Pareto set. Fig. 6 shows the situation as it occurs in the criterion space. It is clearly seen that the Pareto set is non-convex and disconnected. Disjoint parts of the Pareto set correspond to different teammates who can receive the pass; this is the result of that the criteria are substantially non-monotonic, multi-modal functions. 


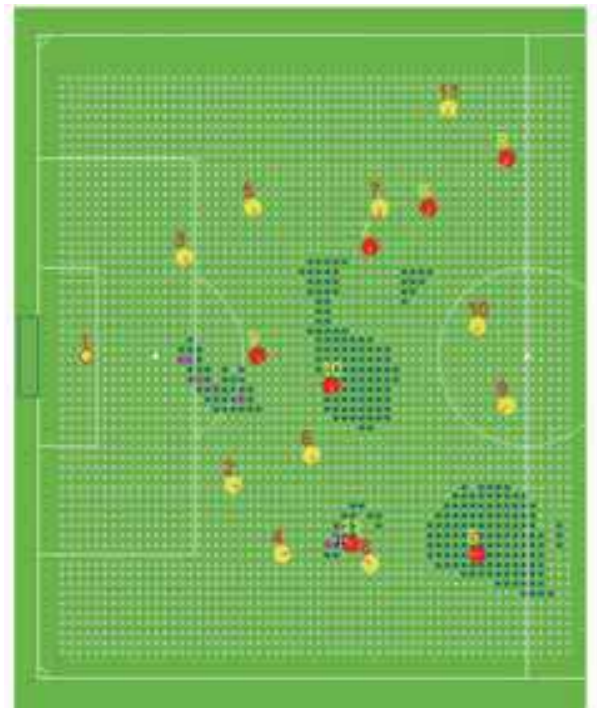

Fig. 5. Situation in Fig.1(d) with the points making the Pareto set highlighted

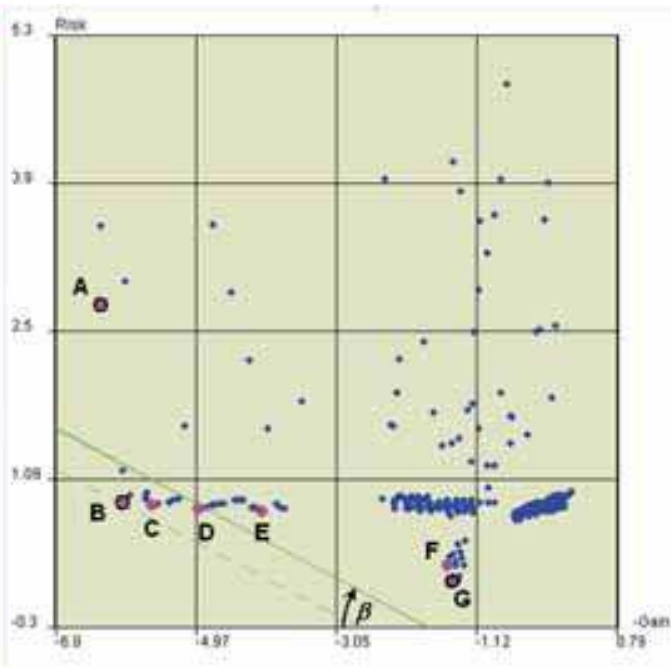

Fig.6. The ball passing alternatives in the criterion space. Points in the Pareto set have larger size

The MCDA theory leaves the final choice of the single alternative from the Pareto set up to the decision maker. In doing so, the latter should rely on his/her aspirations, such as risk taking and risk aversion. In our case, however, the decision maker is the artificial agent; it is the algorithm developer who must formalize the player preferences which could be used for searching the Pareto set for the only 'truly optimal' alternative. This search is exactly about balancing the rewards, risks, and costs; in what follows, we explain the idea of balancing. A naïve approach suggests merging the criteria in just one and applying commonly known single-criterion optimization techniques. For example, one can use the utility function $U$ of the decision variables $(x, y)$, which is a weighted sum of risk Risk and gain Gain:

$$
U(x, y)=-w \cdot \operatorname{Gain}(x, y)+(1-w) \cdot \operatorname{Risk}(x, y),
$$

where $w$ is the positive weight, $0 \leq w \leq 1$; it reflects the importance of Gain for the decision maker, as compared to Risk whose weight is thus $1-w$. (Note the minus sign indicating that we are using -Gain $(x, y)$ as criterion).

To find the solution, the utility function (3) must be minimized. Equation $U(x, y)=c$, where $c$ is some constant, in the criterion space represents the slant straight line shown in Fig.6. Search for the optimal solution in this case would be moving this line towards the origin by decrementing $c$ until the line (shown in the dashed style) intersects with just one decision alternative B. Presumably, this would be the optimal, balanced solution sought.

Unfortunately, this simple approach does work only when the Pareto set is convex [6]. If non-convexity is in place, some elements of the Pareto set would be never rendered as the solutions to the optimization problem, no matter what values the decision maker assigns to $w$. However, this is counter intuitive, as each point in the Pareto set is the best option for some combination of the decision maker preferences. In our example we can scan all possible preferences by making the weight $w$ taking all possible values in the range $0 \leq w \leq 1$. Note 
that, as $\beta=\tan (w)$, this parameter determines the angle $\beta$ of the line $U(x, y)=c$ (in Fig.6, $\mathrm{w}=0.335)$. With $w$ changing, the line is inclined in all the range of angles from 0 to 90 degrees. This 'optimization' process would render only three points, A, B, and G of total seven available in the Pareto set (marked with black circles). The rest four would be never returned as solutions, though, in spite of that the weight is continuously running in the whole permissible range and the missed points are lying in between.

Other known methods based on merging criteria in a single one would be also unsuccessful in the general case if the Pareto set is non-convex. This just illustrates the fact that with the multi-modal criteria functions which we are dealing with in the robotic soccer, a different way to finding the balanced solution of the optimization problem should be taken.

\section{Searching the Optimal Ball Passing Decision in the Pareto Set}

The different way is applying more sophisticated methods for searching the Pareto set that can work with non-convex problems. As there is a plethora of such methods, we will demonstrate just one, developed by the author of this paper. The method is called 'the sequential elimination of the poorest alternative'. Because it does not rely on any information about the criteria functions, it is applicable to any MCDA problem with a finite Pareto set. This nicety, comes at rather low cost: with the total of $K$ elements in the Pareto set, the computational complexity of this algorithm is $O\left(K^{2}\right)$. (Note that $K<<N$, where $N$ is the number of points in the set of the alternatives before any eliminations.)

The key assumption is that each criterion has its relative weight; in our case this information is reflecting the preferences of the developer of the decision making algorithm. So let $\mathbf{X}$ be the set of all alternatives, $\mathrm{P} \subset \mathbf{X}$ be the Pareto set, $\mathbf{x} \in \mathbf{X}$ be a decision vector, $g_{1}(\mathbf{x}), \ldots \mathbf{g}_{\mathrm{n}}(\mathbf{x})$ be the criteria functions (all of which we want to simultaneously minimize), and $w_{1}, \ldots, w_{\mathrm{n}}$ be the non-negative weights whose sum is 1. The algorithm is shown in Fig.7.

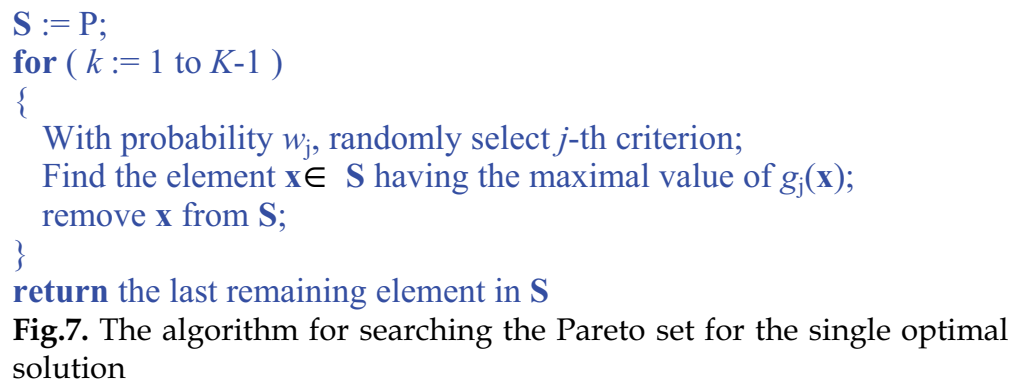

The algorithm has $\mathrm{K}-1$ iterations, eliminating one element from the Pareto at a time. In each step, one criterion is randomly selected; let $j$ be its index. Because weights are used as the probability distribution, more important criteria are being chosen more frequently than the less important ones. For $j$-th criterion, the alternative having the maximal (i.e. the poorest) value of $g_{j}(\mathbf{x})$ is removed from the working copy of the Pareto set $\mathbf{S}$. The process ends when only one element remains in the working copy. This is the approximation of the balanced, optimal solution to the problem. With $K$ increasing, this approximation converges to the precise optimum. 
A simplified version of this algorithm with equal weights does not even require any randomization; criteria for the element removal can be selected in turns. If applied to the example in Fig.6, this simplified method will be first using the -Gain criterion to remove point G. On the next iteration point $\mathbf{A}$ will be removed using Risk. Next $\mathbf{F}$ will be removed using Gain, and so on. The sole surviving alternative in this case will be $\mathbf{D}$, which is the solution. The too scarce discrete subset of the continuous Pareto set like shown in Fig.6 appears to be too rough approximation. Recall that this is all what has remained from the original 3600 points; so if we want a denser approximation of the Pareto frontier, we should be starting with even larger number. Because further increasing this number is not an option, we are using the $\varepsilon$-dominance relation instead of the strict one (Fig.4). This concession can be justified by that the criteria values are calculated with some errors anyway. As we can guesstimate the standard deviation of these errors, we can choose $\varepsilon$ of the same order of magnitude. Thus the discrete $\varepsilon$-Pareto set will be much denser; the application of the random elimination in this case would result in much smaller volatility of the solution.

Fig. 8 and 9 give the idea of what happens to the situation in Fig. 5 and 6 once $\varepsilon$-dominance is applied; the player indeed gets much more options to chose from. The cost for this is slight deviation from the strict Pareto optimality and a longer, yet not prohibitive, computation time. The benefit is the better robustness of the solution search algorithm.

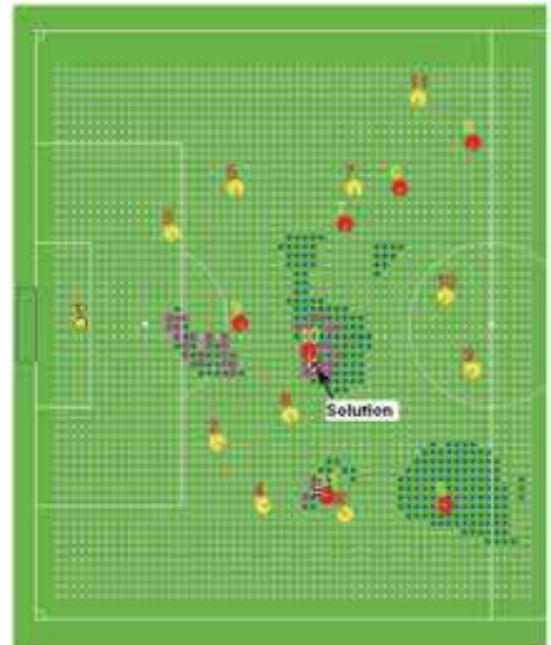

Fig.8. Situation with the $\varepsilon$-Pareto set. Note the increased number of points.

The solution is shown as a black cross. Player prefers avoiding risky passes

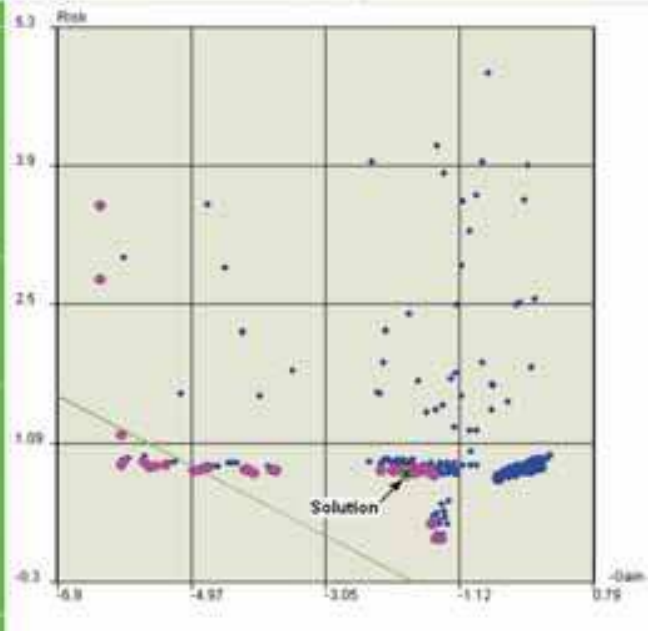

Fig.9. The ball passing alternatives in the criterion space. Points in the $\varepsilon$-Pareto set have larger size. The solution is shown as a black cross. The -Gain weight is 0.335

Assigning weights to the criteria in the proposed framework has transparent meaning. Unlike using weights to sum up criteria similar to (3), in our method there is no way for that a high value of one criterion apparently compensates for the insufficient value of the other. This is because weights determine only the priorities in the elimination of poor alternatives. So it is safe to say that the proposed technique allows easily modeling the full spectrum of 
risk taking and risk aversive attitudes of the decision maker. This is made possible by assigning weights.

So far we have been using the example with the weight of -Gain 0.335 , i.e. Risk had twice as much higher weight. This results in the risk-aversive decision shown in Fig. 8 and 9. Player 11 prefers to pass the ball to teammate 10 rather than taking the chance of sending the ball to teammate 9 whose position appears to be is much better. By changing the weight in favor of higher gains, it is possible to persuade the player to pass the ball to player 9 (see Fig. 10, 11). The slant straight lines shown in Fig. 9 and 11 indicate the weight $w$ only; they are not used to find the solution, as different procedure applies.

\section{Addressing the Real-Time Constraints}

As described so far, the optimal ball passing decision making algorithm in terms of computations appears to be even more demanding than the algorithms proposed in [1,2, and 3]. In the first experiments in 2003 with our simulated soccer team SFUnleashed we have indeed found that the quality of decisions made by players while passing the ball nonmonotonically depended on the total number of points $N$. Starting with small number of points, quality was noticeably increasing with $N$. Then, with greater $N$, we observed significantly decreased performance. Indeed, with large $N$ the player process could not complete all required computations during one simulation cycle.

As it should be expected for a real-time system like robotic soccer, attempts to utilize all the player potential by using sophisticated optimization may be counterproductive because of the prohibitive computation time. Still we decided to find a way out so that the real-time constraints were not so restrictive. Our solution comprises two ways for the time reduction. The first way is further reducing the number of alternatives that wittingly are not in the Pareto set; this can be done by applying more heuristics. Besides those outlined in Fig.1, we are using one more, by replacing the equidistant grid with randomly scattered points in the vicinity of each teammate. In doing so, we want to ensure that the teammate can reach the given point before the ball arrives in it. This is achieved by determining the size of the area around the teammate; this area is then randomly populated by the points (Fig.10, 11).

The second way is automatically adjusting the number of generated points $N$ during the run time with respect to the actually available time in the simulation cycle. As we know that the complexity of the whole method is $O\left(N^{2}\right)$, it is always possible to estimate affordable $N$ in advance in the current simulation cycle and thus prevent real-time constraints from kicking in. Reducing $N$ would result in only gradual increase of the random deviations from the optimal solutions, without any abrupt losses in the quality of decisions on the average. This behavior is quite different from that of the algorithms based on decision trees whose realtime scalability is very limited.

Thus the proposed algorithm is robust by design and it is indeed scalable with respect to tightened or relaxed real-time constraints.

\section{Conclusion}

The full-blown algorithm is just a straightforward generalization of the simplified method illustrated in the above examples. The only difference is that instead of the two criteria function we are using all ten. The algorithms for computing these criteria have been described in Section 2; some of them are similar to that can be found in the RoboCup literature. More 
elaborated criteria set combining different rewards, risks, and costs allows coupling our method with the 'smart' receiving player behavior when the ball is intercepted in the point having best tactical value rather then just in the fastest way. One new criterion allows avoiding failed passes that are possible in the previous algorithm when the receiving player is exhausted. The proposed method is general, as it is equally treating direct and leading passes and passes to self.

Still the major new element of our ball passing algorithm is the application of the Pareto optimality principle and the related method for searching the Pareto set. This innovation guarantees that, for given set of the decision maker preferences, with the discrete approximation of the Pareto set dense enough, the solution of the ball passing problem yielded by this method cannot be further improved. Therefore, the proposed method can be used as a benchmark for evaluating different algorithms based on heuristics.

Our method provides a convenient way to implementing all the range of the decision maker attitudes by balancing gains, risks, and costs in a flexible way. Therefore, the new approach allows enriching the soccer team tactics without complicating the control logic.

The proposed algorithm is robust with respect to the real-time constraints. It allows avoiding the violation of these constraints by adjusting during the run time just one parameter, the total number of decision alternatives in the initial set. As this set is generated randomly, its size reduction would only result in a gradual random deviation from the optimal decision.

As the proposed approach to optimization is general, besides ball passing, we consider using it for optimizing other low-level decision making in the robotic soccer, such as player positioning, dribbling, and scoring the goal. We also expect that similar approach is applicable to higher-level decisions involving the choice between the lower-level actions.

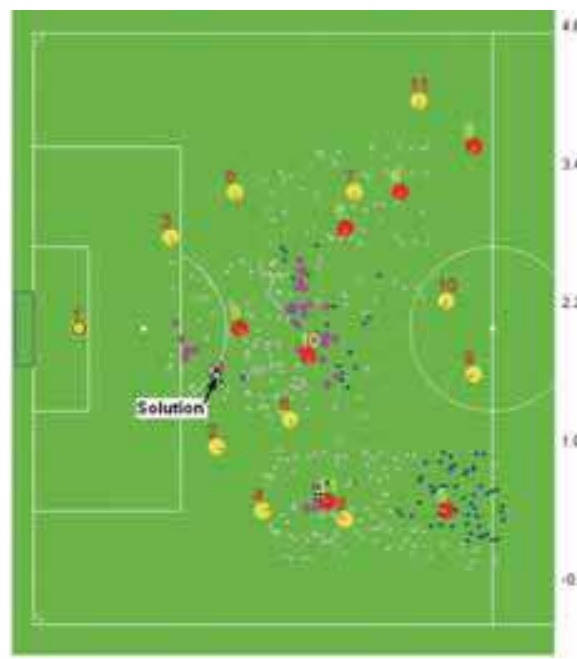

Fig. 10. Situation with 400 points randomly generated about the teammates. The decision is more risky than in Fig. 7., but its gain is higher

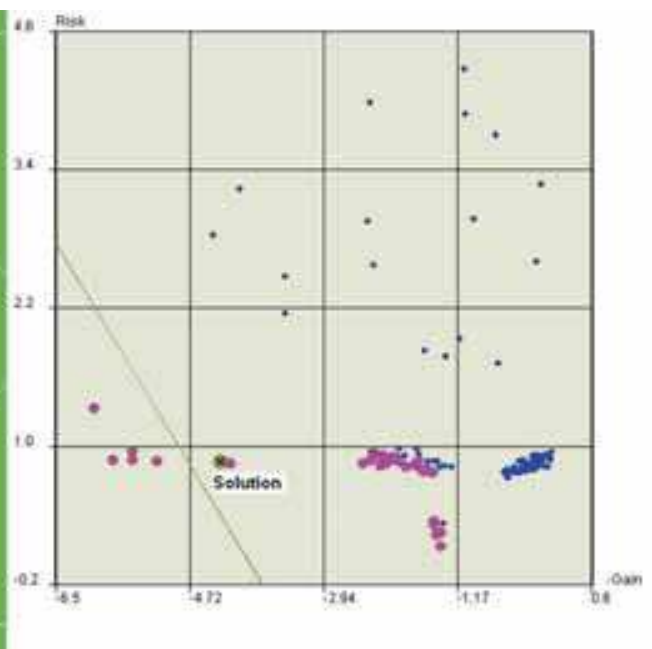

Fig. 11. The ball passing alternatives in the criterion space with $\mathcal{E}$-Pareto set highlighted. The -Gain weight is 0.614 . Note the different angle of the line 


\section{References}

1. Stone, P.; McAllester, D.: An Architecture for Action Selection in Robotic Soccer. In: Proceedings AGENTS'01, 5th International Conference on Autonomous Agents, May 28-June 1, 2001, Montreal, Quebec, Canada. (2001) 316-323

2. Reis, L. P.; Lau, N.: FC Portugal Team Description: RoboCup 2000 Simulation League Champion. In: Stone, P.; Balch, T., and Kraetzschmar, G. (eds.): RoboCup 2000, LNAI 2019. Springer: Berlin Heidelberg New York (2001) 29-40

3. Wang, C.; Chen, X.; Zhao, X. \& Ju, S.: Design and Implementation of a General Decisionmaking Model in RoboCup Simulation, pp., International Journal of Advanced Robotic Systems, 1, No. 3 (2004) 207-212

4. Dylla, F.; Ferrein, A.; Lakemeyer, G. ; Murray, J.; Obst, O.; Rofer, T.; Stolzenburg, F.; Visser, U. and Wagner, T.: Towards a League-Independent Qualitative Soccer Theory for RoboCup. In: Nardi, D. et al. (eds.): RoboCup 2004, LNAI 3276. Springer: Berlin Heidelberg New York (2005) 29-40

5. Ehrgott, M.: Multicriteria Optimization. 2nd ed. Springer: Berlin Heidelberg New York (2005)

6. Jahn, J.: Vector Optimization. 2nd ed. Springer: Berlin Heidelberg New York (2004) 


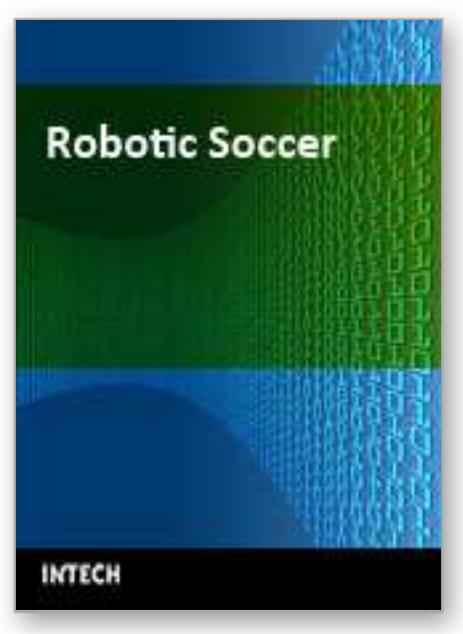

\author{
Robotic Soccer \\ Edited by Pedro Lima
}

ISBN 978-3-902613-21-9

Hard cover, 598 pages

Publisher I-Tech Education and Publishing

Published online 01, December, 2007

Published in print edition December, 2007

Many papers in the book concern advanced research on (multi-)robot subsystems, naturally motivated by the challenges posed by robot soccer, but certainly applicable to other domains: reasoning, multi-criteria decisionmaking, behavior and team coordination, cooperative perception, localization, mobility systems (namely omnidirectional wheeled motion, as well as quadruped and biped locomotion, all strongly developed within RoboCup), and even a couple of papers on a topic apparently solved before Soccer Robotics - color segmentation - but for which several new algorithms were introduced since the mid-nineties by researchers on the field, to solve dynamic illumination and fast color segmentation problems, among others. This book is certainly a small sample of the research activity on Soccer Robotics going on around the globe as you read it, but it surely covers a good deal of what has been done in the field recently, and as such it works as a valuable source for researchers interested in the involved subjects, whether they are currently "soccer roboticists" or not.

\title{
How to reference
}

In order to correctly reference this scholarly work, feel free to copy and paste the following:

Vadim Kyrylov (2007). A Robust and Scalable Pareto Optimal Ball Passing Algorithm for the Robotic Soccer, Robotic Soccer, Pedro Lima (Ed.), ISBN: 978-3-902613-21-9, InTech, Available from: http://www.intechopen.com/books/robotic_soccer/a_robust_and_scalable_pareto_optimal_ball_passing_algorit $\mathrm{hm}$ _for_the_robotic_soccer

\section{INTECH}

open science | open minds

\author{
InTech Europe \\ University Campus STeP Ri \\ Slavka Krautzeka 83/A \\ 51000 Rijeka, Croatia \\ Phone: +385 (51) 770447 \\ Fax: +385 (51) 686166 \\ www.intechopen.com
}

\author{
InTech China \\ Unit 405, Office Block, Hotel Equatorial Shanghai \\ No.65, Yan An Road (West), Shanghai, 200040, China \\ 中国上海市延安西路65号上海国际贵都大饭店办公楼405单元 \\ Phone: +86-21-62489820 \\ Fax: $+86-21-62489821$
}


(C) 2007 The Author(s). Licensee IntechOpen. This chapter is distributed under the terms of the Creative Commons Attribution-NonCommercial-ShareAlike-3.0 License, which permits use, distribution and reproduction for non-commercial purposes, provided the original is properly cited and derivative works building on this content are distributed under the same license. 